\title{
ACCIONES Y RESULTADOS Una propuesta de clasificación de las consecuencias no intencionales
}

\author{
ACTIONS AND RESULTS \\ A Proposal to Classify Unintended Consequences
}

JaVIer L. Cristiano javier.cristiano.m@gmail.com
CONICET, Universidad Nacional de Córdoba. Argentina

\begin{abstract}
RESUMEN
La intención de este artículo es proporcionar una herramienta analítica para teorizar sobre las consecuencias no intencionales de la acción social. La propuesta parte de los pocos trabajos similares que ya existen, analizándolos y reuniéndolos en una única clasificación. En segundo lugar se elaboran distinciones sobre aspectos no tratados por esas clasificaciones; en particular, sobre la dinámica interna de las interacciones que conducen a consecuencias no intencionales. Por último, se ilustran posibles derivaciones de la propuesta conjunta para tres importantes campos de investigación.
\end{abstract}

\section{Palabras Clave}

Acción social, Cambio social, Interdependencia, Responsabilidad.

\begin{abstract}
The intention of this article is to supply an analytical instrument to theorize on the unintended consequences of social action. The proposal is based on the few existing similar works, analyzes them and combines them in a sole classification. Secondly, it develops some distinctions on aspects not considered by those previous classifications; in particular, it focuses on the internal dynamic of interactions that lead to unintended consequences. Finally, the article illustrates possible outcomes of the combined proposal for relevant research fields.
\end{abstract}

\section{KEYWORDS}

Interdependence, Responsibility, Social Action, Social Change. 


\section{INTRODUCCIÓN}

No es éste el lugar para discutir la pertinencia y la importancia de las clasificaciones en la ciencia social. Daré simplemente por sentado que la elaboración de tipologías es una tarea importante y, para muchos fines teóricos y empíricos, indispensable. Pero también supondré que se trata de una tarea abierta y preliminar: toda clasificación depende de criterios de observación que pueden discutirse y modificarse, y se limita a la mera distinción y organización de elementos, como paso previo, y nunca concluyente, a la investigación de un fenómeno.

Hago esta aclaración para fijar de entrada los límites de este artículo. Me propongo un modesto trabajo analítico de depuración y precisión, con la esperanza de proporcionar un mejor sustento para la teorización de un tema crucial. Los elementos que utilizo son propuestas similares, información empírica y teoría propiamente dicha. Pero concibo el trabajo como un esfuerzo de organización y precisión, que en tal sentido es preparatorio y, a efectos de su validez, condicional.

Mi primer paso (apartado 1) será contextualizar la discusión, con una nota sobre la genealogía y la importancia del tema -el de las consecuencias "no intencionales" o "no queridas" de la acción social-. El Apartado 2 entra en materia, analizando tres propuestas de clasificación que ya existen, y que en mi opinión pueden unificarse en una sola, que rescata lo esencial de cada una. Argumentaré sin embargo que todas se refieren a aspectos descriptivos del fenómeno, y deben completarse con distinciones sobre la dimensión interna o "explicativa" (Apartado 3). Unificando ambas dimensiones en una tipología única es posible esbozar (Apartado 4) líneas de investigación prometedoras y hasta ahora poco desarrolladas.

\section{RETROSPECTIVA E IMPORTANCIA DEL TEMA PARA LA SOCIOLOGÍA}

Para remontarse al origen del problema hay que ubicarse en el contexto de la llustración escocesa, donde autores como A. Smith, J. Millar y A. Ferguson dieron al problema su formulación moderna (Wences 2006; 2008; Lamo de Espinosa 1990, cap. 2) ${ }^{1}$. En contraste con las lecturas contractualistas, que interpretaron el orden social como producto de un pacto voluntario, la llustración escocesa lo concibió como "producto de la acción humana pero no de los designios humanos", según la célebre expresión de Ferguson, puesta en práctica en la descripción de diversos fenómenos, la sociedad civil entre ellos. Según su lectura, la sociedad civil moderna no surge de ningún contrato explícito sino de un proceso objetivo que hace uso de las acciones de los individuos pero se desentiende (felizmente para Ferguson) de sus intenciones. Lo mismo ocurre con la mano invisible de A. Smith y

\footnotetext{
${ }^{1}$ La genealogía más amplia del tema puede consultarse en Hayek (1978) y Ramos (2002).
} 
otro tanto, en un contexto muy próximo, con los vicios privados de B. Mandeville, que a contrapelo de la intuición moralista promueven beneficios públicos.

El carácter objetivista de la idea, directamente inspirada en la ciencia newtoniana (Wences 2008), le dio amplia y rápida cabida en la naciente ciencia social. Diversos aspectos de la obra de Marx (admirador por lo demás de Ferguson) aluden a las consecuencias no intencionales en toda regla, como la explicación de la tasa decreciente de ganancia (producto involuntario del perfeccionamiento de la explotación por cada capitalista) o la misma cosificación, resultante de una multitud de acciones que se extrañan de sus consecuencias. Max Weber concibió a la historia como el reino de la "heterogonía de los fines" (Stark 1971), y como es sabido su sociología de la religión abunda en la exposición de consecuencias no queridas (Cherkaoui 2007), empezando por la paradójica relación de la ética protestante con el capitalismo.

Pero lejos de agotarse en su etapa fundacional, el tema que nos ocupa ha incidido en numerosos y fundamentales debates de la teoría social contemporánea ${ }^{2}$. A riesgo de simplificar, pero para dar cuenta clara y brevemente de lo esencial del problema, puede decirse que las consecuencias no intencionales están implicadas en la discusión de por lo menos: el objeto de la sociología (1), los problemas del orden y del cambio social (2), el estatuto metodológico de la disciplina y la naturaleza de la explicación social (3).

1. Respecto de lo primero, son muchos los autores que han sostenido que las consecuencias no intencionales no son un tema entre otros, sino "el" tema de la sociología. Se argumenta -lo ha hecho clásicamente Popper (1957:295)- que si es necesaria la ciencia social es precisamente porque algunos fenómenos (la mayoría de los que interesan) no se explican por la simple voluntad de las personas, exigiendo el estudio objetivo de los actos y de sus consecuencias. Boudon (1981:94 y ss.; 1986) ha defendido la misma idea en forma más matizada y más precisa, y muchos otros autores, sin abrir juicio sobre el deber ser, han centrado de hecho su interés en este tipo de fenómeno (Elias 1982, cap. 3; Schelling 1990). En la medida en que se acepte un concreto postulado ontológico (que la acción humana es el input de los fenómenos sociales), y en la medida en que se admita que la complejidad social desborda por lo general las intenciones, las consecuencias no intencionales definen ipso facto el territorio mismo de la sociología.

2. Pero dentro de ese territorio hay mucho, incluidos los temas clásicos del "orden" y del "cambio" social. Respecto del cambio la razón es evidente, pues al menos una parte de las consecuencias no queridas afectan los órdenes institucionales, implicando una transformación que no surge del objetivo de nadie-aunque en general

\footnotetext{
${ }^{2}$ No es posible hacer una lista exhaustiva de autores y contextos, pero merece destacarse, por su carácter pionero, el artículo "Las consecuencias imprevistas de la acción social" de R. Merton, que dio entidad teórica explícita al tema (1980 y 1989, para una visión retrospectiva).
} 
se acerque al interés de algunos. A esto hay que añadir que la existencia misma de emergentes involuntarios es un importante incentivo para procesos de cambio (ahora sí deliberados), como lo han mostrado Elster (1991, cap. 10; 1994a:175 y ss.) y, con un amplio panorama de ejemplos, Boudon (1980:17 y ss.). Respecto del "orden" la conexión es menos obvia, porque las consecuencias no intencionales parecen explicar mejor el cambio que la cristalización de instituciones. Están ahí sin embargo las "funciones latentes" de Merton, las consecuencias no intencionales que se insertan en circuitos de reproducción, expresamente destacadas por A. Giddens (1995:50-51), la estabilización de pautas de comportamiento como producto de la imitación masiva (Dupuy 1999; Granovetter 1991) y hasta los sistemas sociales en la definición de Habermas -"regulación no normativa de decisiones particulares que se sitúan allende la conciencia de los actores" (1988:167)-. Si bien alejadas de su inicial metafísica optimista, providencialista o liberal, las consecuencias no intencionales continuaron siendo una llave de acceso al "problema del orden".

3. Todo lo anterior conduce a la epistemología, terreno en el que han descollado defensas explícitas del enfoque de consecuencias no intencionales. Nozick (1999) llamó precisamente "explicaciones de mano invisible" a las que revelan mecanismos que conectan acciones con consecuencias extrañas para los actores, tomando en préstamo la expresión de Smith pero librándola también él de resonancias optimistas. La mano invisible puede actuar para bien o para mal, pero señala para Nozick el ideal epistemológico de la explicación social: un estado de cosas emerge como explanandum a partir de la mostración del vínculo que conduce de las acciones a los resultados. Este tipo de explicación ha sido llamado también "explicación por mecanismos" (Hedström 2006), y aunque no todos los partidarios de las consecuencias no intencionales lo suscriben, representa posiblemente al paradigma en su forma más pura. Frente a la explicación simplemente intencional, las explicaciones por mecanismos afirman que las intenciones son una parte de la explicación, de indudable importancia (puesto que explican por qué los actores hacen lo que hacen) pero inevitablemente parcial. Frente a la explicación funcional, que pretende explicar los fenómenos a partir de sus consecuencias benéficas (Cohen 1986:275 y ss.), afirma que ello sólo puede ocurrir si las consecuencias mantienen la pauta a través de un mecanismo oculto, que es lo que hay que revelar (Elster 1992:53 y ss.). Y frente a la explicación causal de tipo hempeliano, afirma que la escasez (o inexistencia) de leyes generales obedece precisamente a que los hechos sociales surgen de la combinación de mecanismos, y no de regularidades nomológicas. La reducción analítica a mecanismos es sólo una de las estrategias explicativas posibles de las consecuencias no intencionales, pero la relevancia que ha adquirido en el debate reciente (Hedström \& Bearman 2009) da cuenta del peso del problema también en el plano epistemológico. 
Estas rápidas pinceladas hablan por sí solas del interés de precisar la naturaleza y las principales variantes del fenómeno. La discusión que sigue arranca con la propuesta de Boudon, que como acabamos de ver se ubica en primera línea entre los partidarios del modelo ${ }^{3}$.

\section{TRES CLASIFICACIONES DE LO "NO INTENCIONAL"}

De acuerdo con Boudon (1980:12) hay tantos tipos de consecuencias no intencionales como combinaciones posibles de las siguientes variables:

\section{Clasificación 1: R. Boudon}

\begin{tabular}{|l|l|l|}
\hline $\begin{array}{l}\text { Obtención de los objetivos } \\
\text { por los agentes }\end{array}$ & Resultados colectivos & $\begin{array}{l}\text { Implicancias de los resultados } \\
\text { para los actores }\end{array}$ \\
\hline Nadie logra los objetivos & Beneficios colectivos & Referidos a todos \\
\hline $\begin{array}{l}\text { Algunos logran los } \\
\text { objetivos }\end{array}$ & Problemas colectivos & Referidos a algunos \\
\hline Todos logran los objetivos & $\begin{array}{l}\text { Beneficios y problemas } \\
\text { Simultáneamente }\end{array}$ \\
\hline
\end{tabular}

${ }^{3} \mathrm{~A}$ diferencia de Baert y Ramos, los autores de las siguientes clasificaciones, Boudon es un vocero del paradigma a nivel internacional. Merece recordarse por tanto lo principal de su aporte, empezando por las expresiones que emplea como sinónimo: "efectos de agregación", "emergentes", "de composición" o "perversos" (esta última como análoga al principio, después independizada en el sentido de efectos "negativos" o "indeseables"). El estudio de consecuencias no intencionales es para Boudon la clave de la sociología, aunque no asume al respecto una posición normativa. Afirma más bien que la sociología más interesante y exitosa ha sido la que descubre consecuencias no intencionales, aun cuando en muchos casos (Marx por ejemplo) no se use la expresión ni se participe expresamente del paradigma. A su juicio, el estudio de consecuencias no intencionales supone además dos asunciones epistemológicas: un individualismo metodológico moderado, y un racionalismo al que define como "complejo" o "situado". Lo primero significa que la consideración de las acciones singulares es el ideal de la explicación, incluso cuando muchas veces no pueda cumplirse, y el incumplimiento no invalide las conclusiones. La racionalidad compleja significa que los actores tienden a hacer lo mejor para sí mismos, en función de sus intereses y del modo en que perciben su situación. No puede imputarse en consecuencia racionalidad en abstracto, sino siempre en función del contexto y del modo en que lo perciben los actores. Los textos citados en el apartado 2 contienen lo esencial de sus ideas al respecto. Y el artículo de van Parijs (1982) ofrece una precisa discusión en el marco de puntos de vista similares. 
Se trata de una sencilla pero útil descomposición del concepto, que toma en consideración los objetivos de los actores (y por lo tanto la variable "logro" o "fracaso" respecto de esos objetivos), la naturaleza de las consecuencias resultantes (resumida en las opciones "problemas" y "beneficios") y las implicaciones de esas resultantes para los propios actores (pueden afectarlos o serles indiferentes). Ejemplos: en la "mano invisible" de Smith "algunos logran sus objetivos" (Ios ganadores del mercado libre), generando "beneficios colectivos" que, a la larga, son favorables "para todos". En el reverso de la mano invisible, que es la contrafinalidad sartreana (Sartre 1963:466; Elster 1991, cap. 10), algunos logran los objetivos inmediatos (por ejemplo, se apropian de más tierras derribando árboles), con resultantes que son "problemas" colectivos (la desertificación generalizada) que terminan afectando a todos. En el mejor de los mundos imaginables (que puede o no ser "posible", según los casos), todos logran sus objetivos, generando beneficios colectivos que repercuten positivamente para todos. En el peor, nadie logra sus objetivos, generando además problemas colectivos que son tales para todos.

Boudon no se propone una clasificación exhaustiva y hace estas distinciones sólo como introducción al tema. Que yo sepa, Patrick Baert (1991) fue el primero en intentar una propuesta completa con fines expresamente taxonómicos y es referencia obligada para cualquier intento similar:

\section{Clasificación 2: P. Baert}

\begin{tabular}{|l|l|l|l|l|}
\hline Objeto de los efectos & $\begin{array}{l}\text { Valor atribuido } \\
\text { al efecto }\end{array}$ & $\begin{array}{l}\text { Relación con la } \\
\text { intención inicial }\end{array}$ & $\begin{array}{c}\text { Cognoscibilidad / } \\
\text { Anticipabilidad }\end{array}$ & Temporalidad \\
\hline $\begin{array}{l}\text { Individuales (del } \\
\text { agente actuante } \\
\text { o de otros) }\end{array}$ & Deseables & $\begin{array}{l}\text { Que cumplen la } \\
\text { intención inicial }\end{array}$ & Esperados & Sincrónicos \\
\hline $\begin{array}{l}\text { Sociales (sistémicos } \\
\text { o agregados) }\end{array}$ & Neutros & $\begin{array}{l}\text { Que frustran la } \\
\text { intención inicial }\end{array}$ & $\begin{array}{l}\text { Previstos pero } \\
\text { no esperados }\end{array}$ & Diacrónicos \\
& $\begin{array}{l}\text { Que ni frustran } \\
\text { ni cumplen la } \\
\text { intención inicial }\end{array}$ & & \\
\hline
\end{tabular}

Aunque Baert no se remite a él, las tres primeras columnas reproducen con mínimas variantes el cuadro de Boudon. La primera ("objeto de los efectos") vuelve a considerar la localización de las consecuencias (los propios actores o los niveles sociales "macro"); la segunda ("valor atribuido al efecto") alude a su apreciación como deseables, indeseables 0 neutros, con la importante diferencia, respecto de Boudon, de que deja abierta la cuestión del "sujeto" de la valoración (pueden ser los propios actores participantes, pero también un observador externo u otros actores ajenos al proceso); 
la tercera alude al cumplimiento de los objetivos que los actores se proponían, más o menos en consonancia con las distinciones de Boudon. Las novedades están en las dos últimas columnas: la temporalidad (las consecuencias pueden ser sincrónicas respecto de las acciones, o pueden ser diacrónicas, darse en una sucesión temporal más larga); y la posibilidad de anticipar y prever las consecuencias: los actores pueden "saber" que sus acciones van a generar ciertas consecuencias ("esperadas"), pueden anticipar la "posibilidad" de que se produzcan, aunque no las esperen; o pueden, directamente, no concebir siquiera la posibilidad de que ocurran. Ejemplos: el comerciante que en un contexto de inflación eleva sus precios para cubrirse, sabe que casi todos harán lo mismo y que la consecuencia será casi seguro más inflación; el estratega que amenaza a su contrincante (en la diplomacia o en la competencia comercial) sabe que un resultado posible es su retroceso, pero otro el envalentonamiento y la contraofensiva, "consecuencia no intencional" de su amenaza; el individuo que entra en su casa como todas las noches "no sabe" que al encender la luz asustará al ladrón inexperto, que se pondrá nervioso y le disparará antes de huir.

Si el lector se detiene un momento más en estas dos clasificaciones advertirá la ambigüedad que puede encerrar (y de hecho encierra en muchos usos teóricos corrientes) la expresión "no intencional". En verdad, las dos clasificaciones ayudan a desentrañar la ambigüedad, distinguiendo, aunque en trazos todavía gruesos, tres fenómenos distintos: la "intención" propiamente dicha (en el amplio sentido de "lo estados de cosas futuros que los actores quieren producir con su acción"), la deseabilidad de lo que finalmente ha ocurrido como consecuencia de la acción (que puede ser "otra cosa" respecto del objetivo, y como tal "deseable, indeseable o indiferente"), y por último, las "previsiones" que el actor hace y puede hacer en el momento de su acción. Las tres cosas se confunden cuando se habla de consecuencia "no intencional" y son, como es obvio, completamente distintas. La tercera clasificación (Ramos 1993) propone justamente un esquema (página siguiente) para distinguir estos fenómenos.

La taxonomía se basa en tres pares de conceptos: querido/previsto; agrega/reemplaza; previsible/deseable. Lo "querido" es del orden de lo volitivo: lo que los actores desean o buscan; mientras lo "previsto" es, en el orden cognitivo, lo que los actores anticipan que va a ocurrir (más allá de que lo quieran o no). "Agregan" y "reemplazan" se refieren a la relación entre las consecuencias efectivamente ocurridas y lo querido/previsto por los actores. Lo "previsible" es lo que los actores están en condiciones de anticipar (nivel cognitivo), mientras lo "deseable" es lo que valoran positivamente como estado de cosas del mundo (nivel volitivo). Nótese que aquí es más difícil la ejemplificación con conceptos (como la "mano invisible"), porque necesitamos información empírica mucho más detallada. Nótense también, aunque sea al pasar, las sutilezas teóricas a cuya discusión invitan las categorías. Por ejemplo, que lo "previsible" o "imprevisible" de las consecuencias alude tanto a las condiciones sociales de los actores (la velocidad del proceso, por ejemplo, o su complejidad y magnitud, o aspectos culturales específicos, como las creencias y la educación) como a la naturaleza del conocimiento social mismo, incluido el que puede proporcionar la ciencia social. Adviértase, por último, el interés 


\section{Clasificación 3: Ramos}

\begin{tabular}{lll}
\multicolumn{1}{c}{$\begin{array}{l}\text { Intenciones y } \\
\text { previsiones del actor }\end{array}$} & \multicolumn{1}{c}{ Logro / fracaso de los actores } & Previsibilidad / deseabilidad \\
Querida y prevista & Que se agregan a lo querido y previsto & Previsibles y deseables \\
Querida y no prevista & $\begin{array}{l}\text { Que se agregan a lo querido, pero reemplazan } \\
\text { a lo previsto }\end{array}$ & Previsibles, pero indeseables \\
$\begin{array}{ll}\text { No querida, pero } \\
\text { prevista }\end{array}$ & $\begin{array}{l}\text { Que reemplazan a lo querido y se agregan a } \\
\text { lo previsto }\end{array}$ & Imprevisibles, pero deseables \\
$\begin{array}{ll}\text { No querida y no } \\
\text { prevista }\end{array}$ & $\begin{array}{l}\text { Que reemplazan tanto a lo querido como a lo } \\
\text { previsto }\end{array}$ &
\end{tabular}

de esta taxonomía para abordar problemas inmediatamente relevantes de la sociedad contemporánea, como los que describen la "teoría del riesgo" o de la "reflexividad".

Lo esencial para nuestros fines, sin embargo, es que las tres clasificaciones se complementan y se mejoran entre sí. Hasta el punto de que puede elaborarse con ellas -con unos pocos ajustes y simplificaciones- una única clasificación general que, en mi opinión, rescata los aportes esenciales de cada una. He aquí el cuadro de integración:

\section{Clasificación Integrada*}

\begin{tabular}{|c|c|c|c|c|c|}
\hline \multicolumn{2}{|c|}{ Objeto y Naturaleza } & $\begin{array}{l}\text { Intenciones y } \\
\text { previsiones del actor }\end{array}$ & $\begin{array}{l}\text { Logro / fracaso } \\
\text { de los actores }\end{array}$ & $\begin{array}{l}\text { Previsibilidad/ } \\
\text { deseabilidad }\end{array}$ & Temporalidad \\
\hline \multicolumn{2}{|c|}{ Individuales } & \multirow{4}{*}{$\begin{array}{l}Q ; P \\
Q ; \text { no } P \\
\text { no } Q ; P \\
\text { no } Q ; \text { no } P\end{array}$} & \multirow{4}{*}{$\begin{array}{l}+Q ;+P \\
+Q ; r P \\
r Q ;+P \\
r Q ; r P\end{array}$} & \multirow{4}{*}{$\begin{array}{l}\mathrm{Pb} ; \mathrm{D} \\
\mathrm{Pb} ; \text { no D } \\
\text { no } \mathrm{Pb} ; \mathrm{D} \\
\text { no } \mathrm{Pb} ; \text { no D }\end{array}$} & \multirow{4}{*}{$\begin{array}{l}\text { Sincrónicos } \\
\text { Diacrónicos }\end{array}$} \\
\hline \multirow{3}{*}{ Sociales } & & & & & \\
\hline & Problemas & & & & \\
\hline & Beneficios & & & & \\
\hline \multicolumn{2}{|c|}{ Todos / algunos } & Todos / algunos & Todos / algunos & $\begin{array}{l}\text { Todos I } \\
\text { alaunos }\end{array}$ & \\
\hline
\end{tabular}

"Por razones de espacio se han simplificado las distinciones de la clasificación de Ramos: $Q$ = querido; $\mathrm{P}=$ previsto; + = "se agregan"; $\mathrm{r}=$ "reemplazan"; $\mathrm{Pb}=$ previsible; $\mathrm{D}=$ deseable. 
Todo el análisis de Ramos perfecciona y profundiza las columnas 1 y 2 de la clasificación 1 (Boudon), y la 2, 3 y 4 de la clasificación 2 (Baert). Por eso forma el tronco de la clasificación integrada (las tres columnas centrales). La primera columna de la izquierda une aportes de Boudon y Baert respecto de la "naturaleza" de las consecuencias y "del lugar en que ocurren": nivel individual o colectivo y carácter beneficioso o perjudicial en el caso del segundo (consecuencias "sociales"). La quinta columna incorpora la temporalidad de Baert, y la fila inferior incluye, en todos los casos en que es pertinente, el operador cuantificacional "todos/algunos", que aportaba Boudon en su clasificación.

El lector puede proceder por su cuenta en la ejemplificación de los matices. Va de suyo que las categorías del cuadro no lo abarcan todo (tampoco lo pretenden), pero también es obvio que precisan y diferencian muchos aspectos relevantes del fenómeno. La noción de "consecuencias no intencionales" como tal adquiere, a la luz de la clasificación, una precisión categorial que en su uso corriente no tiene y de la que pueden hacerse diversos usos totales o parciales. Sin olvidar lo dicho al principio sobre el carácter preliminar de cualquier trabajo taxonómico, el resultado es un importante paso de síntesis.

\section{LOS ASPECTOS "INTERNOS"}

La clasificación aborda, sin embargo, sólo el aspecto externo del fenómeno. Nos dice genéricamente "qué son" las consecuencias no intencionales, cuáles son sus principales manifestaciones, pero aporta poco al esclarecimiento de cómo se producen, esto es, cómo se disponen acciones e interacciones para dar lugar a resultados no intencionales. Este otro aspecto -que propongo llamar "interno" o "explicativo" 4 - trasciende por completo un esfuerzo sólo taxonómico, pero requiere también distinciones y matices. Puesto que es un ámbito mucho más complejo, la propuesta que sigue es abierta y conjetural con mucha más razón que la anterior.

Los fenómenos de consecuencias no intencionales abarcan, en primer lugar, dos cosas sustantivamente distintas: los efectos directos que los actores producen con su acción (en una relación que, para los fines presentes, puede entenderse como de causaefecto ${ }^{6}$ ), y los efectos que su acción contribuye a producir debido a la combinación con otras acciones.

\footnotetext{
${ }^{4}$ La palabra "explicativo" se emplea aquí en el sentido más general de "respuesta a las preguntas por qué y cómo" ocurre un fenómeno o estado de cosas (en este caso, una "consecuencia no intencional").

${ }^{5}$ La clasificación retoma parcialmente la que propuse en otro trabajo, dedicado a los "efectos perversos" como tipo específico de "consecuencia no intencional" (Cristiano 2004:216 y ss.). El cuadro que cierra este apartado es la guía para seguir el análisis.

6 "Causa- efecto" en el sentido simple de una relación inmediata, temporal y espacialmente acotada, entre la acción y su consecuencia.
} 
1. Considérese, como ilustración de lo primero, el caso de los ahorristas del banco que caen en la "profecía de autocumplimiento" (Merton 1965:420): creen que el banco es insolvente y corren a recuperar su dinero, generando efectivamente la insolvencia, que es una clara "consecuencia no intencional". Consideremos, en concreto, un aspecto de este complejo fenómeno: formalmente, todos los ahorristas ejecutan un mismo acto -ir a recuperar su dinero- cuya consecuencia inmediata es el aumento de la iliquidez del banco, en el sentido de que cada uno hace un aporte directo a la iliquidez, más allá de su cuantía. Para este tipo de fenómenos son especialmente adecuadas las palabras "efecto" o "consecuencia": los resultados surgen directamente de cada una de las acciones individuales implicadas. Propongo llamarles por eso "efectos" no intencionales.

2. El ejemplo del ladrón que vimos más arriba es, a pesar de su simpleza, formalmente muy distinto. Tenemos allí dos actores (el ladrón y su víctima) que ejecutan actos formalmente distintos (encender la luz uno, disparar nervioso y huir, el otro) cuya relación con la resultante (la muerte) es completamente diferente: uno de ellos la provoca directamente con su acto (relación causa-efecto), mientras el otro crea involuntariamente las condiciones para que ocurra. El ejemplo es trivial pero ilustra el escenario más frecuente en los fenómenos reales de consecuencias no intencionales: un conjunto de actos "cualitativamente distintos", forman una "trama" de interacción que da lugar a resultantes involuntarias. Ejemplos más interesantes y complejos son cuantiosos en la investigación sociológica. ${ }^{7}$ Es un segundo tipo general de consecuencia no intencional, y puede llamarse "procesos" no intencionales.

1.a. Los "efectos" no intencionales incluyen al menos dos tipos de fenómenos que también importa distinguir. Por un lado, hay efectos en los que el aporte que hace cada actor a la resultante "es independiente de lo que hagan los demás". El caso de los ahorristas del banco es un ejemplo: "siempre" que alguien retira sus ahorros disminuye la liquidez del banco, aunque sea en forma infinitesimal. Lo mismo ocurre con las estadísticas de simple agregación, como las tasas de natalidad o los índices de precios (cada comerciante que aumenta el precio contribuye a aumentar el índice general, con independencia de lo que hagan los demás). Hay en otras palabras una relación "necesaria" entre el acto y la resultante. Podemos hablar aquí de lógicas de "agregación", porque la consecuencia no intencional es de hecho el "agregado" o suma de los efectos de actos particulares.

1.b. Por contraste, hay fenómenos en los que la consecuencia de la acción de cada uno depende de la acción de los demás, de modo que será una u otra en función de lo que los otros hagan o dejen de hacer. Una ilustración clásica es la del dilema del prisionero, donde "la misma" acción de un actor (por ejemplo, cooperar en la acción colectiva)

\footnotetext{
${ }^{7}$ Por mencionar sólo algunos recientes: Cramer (2005); Linares Martínez (2005); Roots (2004).
} 
genera resultados completamente distintos en función de lo que hagan sus pares. En el ejemplo de la deforestación, la misma acción individual (por ejemplo, la tala de árboles) puede generar o no desertificación, en función de que los otros hagan o no lo mismo hasta llegar a un nivel crítico. La relación entre el acto y su resultado no es aquí necesaria sino contingente: puede ser una u otra en función de otros actos. Por eso podemos llamar a esta lógica "interdependencia".

2.a. A pesar de su complejidad, el campo de los procesos no intencionales permite distinguir también algunas lógicas elementales. En primer lugar, hay procesos sencillamente lineales, donde los actos que conforman la trama se dan en una sucesión temporal. En el ejemplo del ladrón el individuo que llega a su casa ejecuta un acto (encender la luz para instalarse) que crea las condiciones para la acción de otro (el ladrón) que es quien produce la consecuencia no intencional de la acción del primero (el asesinato). Las investigaciones clásicas sobre knowledge gap han demostrado por ejemplo que la difusión de programas educativos destinados a igualar oportunidades suele tener efectos contrarios, porque la desigualdad preexistente incluye disposiciones también desiguales para asimilar y usar la nueva información. El aumento de la desigualdad es una consecuencia no intencional de la difusión de los programas, en una lógica lineal que formalmente equivalente a la del ladrón.

Un importante matiz de estos procesos lineales es que no siempre incluyen encadenamientos causales, como en los ejemplos anteriores. La sucesión temporal puede estar dominada también por la "casualidad", como ha mostrado Elster con su conocido análisis de la injusticia que surge de decisiones justas (Elster 1994b): un individuo tiene la mala suerte de reunir los atributos que las instituciones desmerecen en el reparto de bienes públicos (tiene demasiada edad para obtener becas de estudio, es demasiado saludable aún para recibir el transplante que de todos modos es inevitable, es elegido en la reducción de personal por no tener hijos a cargo, etc.). Las sucesivas decisiones se propusieron ser justas y en sí mismas lo son, pero producen sumadas, fortuitamente, la consecuencia no intencional de una injusticia flagrante. Nótese de paso que esta "casualidad" es formalmente distinta, a su vez, de la que describe el "efecto mariposa", donde lo fortuito son los sucesivos pasos del encadenamiento causal.

2.b. Junto a las lógicas de sucesión tenemos el importante género de los procesos no intencionales "circulares", tema éste (la circularidad) que ha ocupado también la atención de muchos analistas (Tichenor, Denohue \& Olien 1970) (Myrdal 1957). Baste decir aquí que un proceso circular puro consiste en la relación de mutuo condicionamiento o influencia de al menos dos acciones, de modo que una de ellas ("A") condiciona a la otra ("B"), que condiciona a su vez a la primera ("A"). En el caso de "procesos no intencionales", una de las acciones, o ambas combinadas, producen la consecuencia no intencional ("C"): 


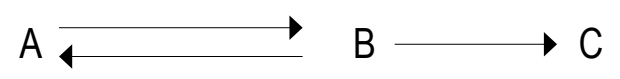

El conocido análisis de Merton del racismo sindical (Merton 1965:421) ilustra esta lógica en un proceso social complejo: los sindicatos racistas tienen el control del acceso al mercado laboral, y discriminan a los trabajadores negros por considerarlos sindicalmente desleales. De este modo crean las condiciones para que los negros acepten trabajar irregularmente en días de huelga, con lo que la opinión de los sindicalistas se refuerza y las medidas discriminatorias se acentúan, afianzando el círculo. En forma un tanto paradójica, el refuerzo del prejuicio, y de la consiguiente desigualdad y discriminación, es una consecuencia no intencional de la acción de los propios sindicalistas.

La estabilidad y resistencia es la principal característica de los procesos circulares, pero no siempre se limita a la permanencia del proceso en el tiempo: a veces conlleva un aumento en la intensidad, volumen o cuantía de las consecuencias, según una lógica de "proceso en espiral" que tiene mucho interés destacar. El ejemplo de Merton sirve también para ilustrarlo, porque los prejuicios raciales se refuerzan y arraigan con cada vuelta del proceso, dando lugar a medidas cada vez más restrictivas y discriminatorias que fortalecen a su vez el proceso conjunto. No hace falta extenderse sobre la importancia teórica y práctica de este matiz, que puede apreciarse en fenómenos como el pánico (Dupuy 1999), los desequilibrios bursátiles o la hiperinflación.

2.c. Finalmente, los procesos no intencionales pueden tener una forma más complejamente reticular, donde no es posible establecer linealidades ni círculos puros porque las acciones se producen tanto en sucesión como en simultaneidad. En otras palabras, conforman una red de la que emergen consecuencias no intencionales. Las investigaciones de newsmaking (Fishman 1979) en teoría de la comunicación han mostrado por ejemplo de qué modo la producción de noticias "ideológicas" (en el sentido de no-objetivas y funcionales a los intereses del poder) es con frecuencia producto no buscado de una compleja amalgama de acciones rutinizadas de periodistas, directivos de medios y portavoces de fuentes burocráticas, orientadas todas ellas según criterios particulares pero productoras, en conjunto, de una distorsión involuntaria respecto de los hechos reales.

3. Los últimos ejemplos llaman la atención sobre una distinción básica más: hay consecuencias no intencionales que se hallan institucionalizadas -en el sentido de que son procesos estables, que se repiten en el tiempo y forman parte de la estructura de una situación- y consecuencias que tienen la forma de acontecimientos, evanescentes en el tiempo. No hay ninguna razón para suponer a priori que las primeras sean más importantes que las segundas, aunque es cierto que la sociología teórica se ha preocupado mucho más intensamente por ellas. En cualquier caso, todos los tipos de efectos y procesos (agregaciones e interdependencias; cadenas, círculos y redes) admiten formalmente ambas manifestaciones. 
Consecuencias no intencionales. Aspectos internos.

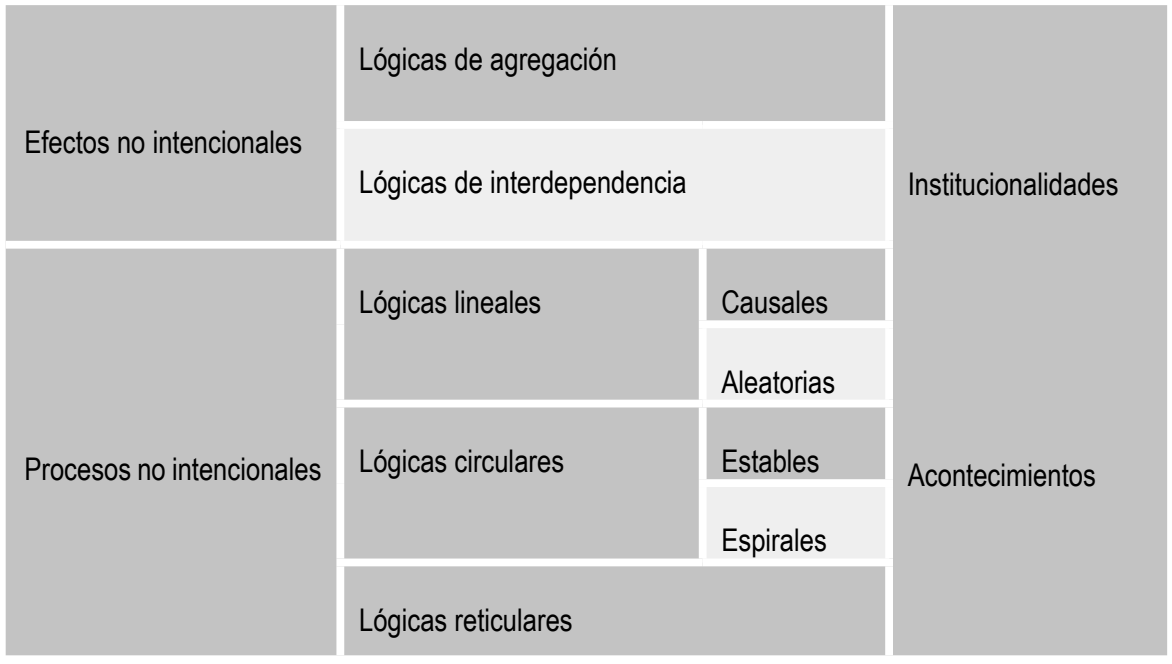

Este conjunto de distinciones básicas no pretende, como es lógico, agotar los matices del aspecto interno. Guarda silencio sobre muchos temas que merecen más detalle -por ejemplo, los múltiples sentidos en que puede decirse que una acción crea las condiciones para otras- y no incluye muchas otras variables posibles -que dependen como es lógico de las demandas teóricas y empíricas de cada investigación concreta. El punto de partida que proporcionan, sin embargo, es más que suficiente como instrumento de refinamiento mental para pensar los problemas teóricos en los que se implican consecuencias no intencionales. Si se colocan juntas, esta clasificación interna y la externa formulada al final del apartado 2 , contamos con una grilla bien dispuesta para sopesar con claridad de qué estamos hablando (o decidimos hablar) cuando decimos consecuencia no intencional.

\section{CoNCLUSIÓN PROSPECTIVA: TRES CAMPOS PARA NUEVAS INVESTIGACIONES}

Una utilidad de la clasificación es, en efecto, la discusión retrospectiva de argumentos. Por ejemplo, cuando Giddens sostiene la importancia de las consecuencias no intencionales para explicar la reproducción estructural, parece claro que está pensando en ciertos tipos de consecuencias (sobre todo, de "procesos circulares institucionalizados"), o por lo menos, que el sentido y calado de su afirmación resulta muy distinto según lo refiramos 
a uno u otro tipo. En este apartado final me interesa destacar la otra utilidad posible, obviamente ligada a ésta, que es perfilar campos de investigación. Señalaré tres en forma muy sucinta, sólo a título de ilustración y sin ninguna pretensión de exhaustividad.

1. El primero es, sencillamente, la continuidad teórica de la tarea de matización. La línea seguida hasta aquí es analítica en el sentido de que busca eliminar ambigüedades relacionadas con el lenguaje ordinario y la lógica elemental. Pero permite continuar el trabajo en un sentido propiamente teórico y, más específicamente, de teoría sociológica. Por ejemplo, las distinciones entre "querido" y "previsto", o las nociones de "cumplimento de la intención" o estado de cosas "deseado", corresponden todas a una visión descarnada (puramente lógica) de la acción humana, y deben "llenarse" con contenidos teóricos que inevitablemente abrirán nuevos matices. Así, una consecuencia no intencional "no querida, no prevista, indeseable e imprevisible", es una realidad analíticamente única en el cuadro, pero teóricamente muy distinta según se la piense, por ejemplo, en clave de "elección racional" o estructura del "mundo de la vida". Este tipo de análisis no corresponde ya al universo de las taxonomías, sino al de la teoría sociológica strictu sensu. Su desarrollo recibe una contribución del trabajo analítico anterior, en el mismo sentido en que cualquier construcción teórica puede orientarse mejor con un análisis del lenguaje ordinario y una mínima depuración lógica.

2. El segundo y muy amplio campo de investigación retoma el vínculo planteado al comienzo entre consecuencias no intencionales y cambio/orden social. Las distinciones del cuadro invitan a reabrirla de un modo que hasta ahora no ha sido muy explorado, puesto que permite plantear con precisión, por ejemplo, la pregunta sobre el tipo de cambio que promueven distintos "tipos" de consecuencias no intencionales. Así, por ejemplo, las consecuencias no intencionales que, para "todos" los actores participantes, son "no queridas ni deseadas, y reemplazan a lo querido y previsto", promueven hipotéticamente un tipo de cambio social "organizativo", mediante esfuerzos de coordinación movidos por el fracaso colectivo y la voluntad de evitarlo a futuro. Cosa muy distinta de lo que ocurre con consecuencias que son indeseables e imprevisibles "para algunos", pero previstas y deseadas para otros. En estos casos tenemos interacciones "contradictorias", promotoras seguramente de cambios sociales "conflictivos". La posibilidad de que el cambio social sea "controlado" por los actores es también muy variable según el tipo de que se trate. Pareciera que cuanto más cerca se esté de los "efectos" no intencionales y de las lógicas de "agregación", más factible será que los actores puedan hacerse con el control de las resultantes. Y a la inversa, cuando más cerca se esté, por ejemplo, de "procesos" no intencionales con lógicas "reticulares", o de procesos "lineales aleatorios", menos campo de acción tendrá el cambio deliberado, y más el cambio no-intencional. Consideraciones análogas pueden hacerse respecto de la estabilización de instituciones y pautas de interacción. Parece lógico pensar por ejemplo que una pauta de interacción en la que "todos" logran sus objetivos y se generan además "beneficios colectivos" que recaen sobre "todos", tenderá a estabilizarse en el tiempo aún cuando los actores no sean 
conscientes del proceso. Algunos tipos de consecuencias no intencionales (las "lógicas circulares estables e institucionalizadas") son además en sí mismas explicaciones del orden social.

Por supuesto, estas sugerencias son verosímiles en el marco de ciertos supuestos teóricos, que deberían discutirse en la investigación concreta. Valen sólo para ilustrar el tipo de pregunta y de indagación que el cuadro promueve.

3. Finalmente: las relaciones entre consecuencias no intencionales y universo prácticomoral. La expresión misma consecuencia no intencional está cargada de resonancias en ese plano. En la sensibilidad propia de la cultura occidental, la ausencia de intencionalidad es un atenuante frente a daños producidos y, al mismo tiempo, el esfuerzo de prever las consecuencias (ensanchar el campo de la conciencia, y por ende de la intencionalidad) es un imperativo moral de amplísimo alcance. Resulta curioso que la sociología, que ha exaltado de tantas formas la cuestión de las consecuencias no intencionales, y que tiene entre sus textos canónicos (por ejemplo) la defensa weberiana de la ética de la responsabilidad en política, no haya hecho esfuerzos más sistemáticos para unir estos dos campos ${ }^{8}$. La taxonomía sugiere un modo en que podría hacerlo. La hipótesis general sería que la acción responsable -entendiendo por tal la acción deliberadamente abocada a cuidar sus consecuencias en consideración del mundo de los valores- tiene condiciones sociales de posibilidad variables, que pueden desentrañarse sociológicamente y de las que son indicio algunas de las distinciones del cuadro. Así, por ejemplo, el problema de lo imprevisible no es solamente de orden lógico o epistémico, sino también social: se vincula al conocimiento práctico de los actores y a la naturaleza de los procesos en que participan (su velocidad y complejidad, por ejemplo). Las lógicas de interdependencia -donde, como vimos, el resultado de la acción depende contingentemente de otras acciones- son una condición social altamente dificultosa para la responsabilidad, lo mismo que los procesos aleatorios que son al mismo tiempo acontecimientos. El lector puede releer el cuadro en esta clave y formular sus propias hipótesis, que de hecho son muchas, y con frecuencia polémicas.

Al hablar de condiciones de posibilidad de la acción responsable tocamos, también, el núcleo político del problema. Por un lado, la búsqueda de formas interacción que hagan más factible la acción responsable puede postularse como un aspecto importante de la agenda política, en tiempos en que la tendencia objetiva (riesgo ecológico, complejidad, etcétera) parece ser precisamente la contraria. Por otro, el discurso político de autojustificación en términos de "opacidad del mundo social" tiene su contrapeso en una sociología rigurosa de las consecuencias no intencionales.

\footnotetext{
${ }^{8}$ Vale recordar aquí el texto, en este sentido seminal, de Giner (2008), y también las incursiones que en la misma dirección ha hecho Bauman (1998:229).
} 


\section{REFERENCIAS BIBLIOGRÁFICAS}

Baert, Patrick. 1991. "Unintended Consequences: A Typology and Examples." International Sociology 2:201-210.

Bauman, Zygmund. 1989. Modernidad y holocausto. Toledo: Sequitur.

Boudon, Raymond. 1980. Efectos perversos y orden social. México: Premiá.

Boudon, Raymond. 1981. La lógica de lo social. Madrid: Rialp.

Boudon, Raymond. 1986. Theories of Social Change. Oxford: Polity Press.

Cherkaoui, Mohamed. 2007. Good Intentions. Max Weber an the Paradox of the Unintended Consequences. Oxford: The Bardwell Press.

Cramer, Elizabeth. 2005. "Unintended Consequences of Constructing Criminal Justice as a Dominant Paradigm in Understanding and Intervening in Intimate Partner Violence." Women's Studies Quarterly 1-2:272-289.

Cohen, G. 1986. La teoría de la historia de Karl Marx: una defensa. Madrid: Siglo XXI/Pablo Iglesias.

Cristiano, Javier. 2004. "Interacciones perversas. Una reconstrucción del problema de los 'efectos perversos' en sociología", Tesis Doctoral, Universidad Complutense de Madrid: Madrid. (Inédito).

Dupuy, Jean P. 1999. El pánico. Barcelona: Gedisa.

Elias, Norbert. 1982. Sociología fundamental. Barcelona: Gedisa.

Elster, Jon. 1991. Tuercas y tornillos. Barcelona: Gedisa.

Elster, Jon. 1992. El cambio tecnológico. Barcelona: Gedisa.

Elster, Jon. 1994a. Lógica y sociedad. Barcelona: Gedisa.

Elster, Jon. 1994b. Justicia local. Barcelona: Gedisa.

Fishman, Mark. 1979. La fabricación de la noticia. Buenos Aires: Tres Tiempos.

Giddens, Anthony. 1995. La constitución de la sociedad. Buenos Aires: Amorrorn.

Giner, Salvador. 2008. "Sociología y filosofía moral". Pp. 118-162 en Historia de la ética, editado por Victoria Camps. Barcelona: Crítica.

Granovetter, Mark. 1991. "Modelos de umbral de conducta colectiva". Pp. 137-166 en Intereses individuales y acción colectiva, compilado por F. Aguiar. Madrid: Pablo Iglesias.

Habermas, Jürgen. 1988. Teoría de la acción comunicativa Il. Crítica de la razón funcionalista. Madrid: Tecnos. 
Hayek, Fiedrich. 1978. New Studies in Philosophy, Politics, Economics and the History of Ideas. Londres: Routledge and Kegan Paul.

Hedström, Peter. 2006. "Explaining Social Change: An Analytical Approach." Papers 80:73-95.

Hedström, Peter and Peter Bearman. 2009. "What is Analytical Sociology all About? An Introductory Essay". Pp. 3-24 en The Oxford Handboock of Analytical Sociology, edited by Hedströn \& Bearman. Oxford: Oxford University Press.

Lamo de Espinosa, Emilio. 1990. La sociedad reflexiva. Madrid: CIS

Linares Martínez, Francisco. 2005. "Juegos de estrategia y consecuencias inintencionadas: un modelo con resultados perversos de la crisis de la minería de Cartagena-La Unión." Papers 75:35-71.

Merton, Robert. 1965. Teoría y estructura sociales. México: FCE.

Merton, Robert. 1980. Ambivalencia sociológica y otros ensayos. Madrid: Espasa-Calpe.

Merton, Robert. 1989. "Unanticipated Consequences and Kindred Sociological Ideas: A Personal Gloss", Pp. 307-329 en Morgadini, C \& Taboni, S. L'Opera di Robert Merton e la sociologia contemporanea. Genova: EGIG.

Myrdall, Gunar. 1957. Rich Lands and Poor. Nueva York: Harper.

Nozick, Robert. 1999. Puzzles socráticos. Madrid: Cátedra.

Parijs, P. van. 1982. "Perverse Effects and Social Contradictions: Analytical Vindication of Dialectics?." British Journal of Sociology 4:589-603.

Popper, K. 1957. La sociedad abierta y sus enemigos. Buenos Aires: Paidós.

Ramos Torre, Ramón. 1993. "Una aproximación a las paradojas de la acción social". Pp. 435-471 en Problemas de teoría social contemporánea, editado por Emilio Lamo de Espinosa \& José Rodríguez Ibáñez. Madrid: CIS.

Ramos Torre, Ramón. 2002. "Sobre los orígenes del principio consecuencial: Leibniz, Mandeville y Vico". Pp. 1.013-1.025 en La sociedad: teoría e investigación empírica, editado por Julio Iglesias de Usell. Madrid: CIS.

Roots, Roger. 2004. "When Laws Backfire: Unintended Consequences of Public Policy." American Behavioral Scientist 11:1376-1394.

Sartre, J. P. 1963. Crítica de la razón dialéctica. Buenos Aires: Losada.

Schelling, Thomas. 1990. Micromotivos y macroconducta. México: FCE.

Stark, Wernerk. 1971. "Max Weber y la heterogonía de los fines". Pp. 191-208 en Presencia de Max Weber, compilado por Talcott Parsons. Buenos Aires: Nueva Visión.

Tichenor, D., G. Denohue \& C. Olien. 1970. "Mass media and diferencial Grown in Knowledge." Public Opinion Quaterly 34:158-70. 
Wences, María Isabel. 2006. Sociedad civil y virtud cívica en Adam Ferguson. Madrid: Centro de Estudios Constitucionales.

Wences, Maria Isabel. 2008. "La relevancia sociológica de la ilustración escocesa." Revista Internacional de Sociología 68:37-56.

JAVIER L. CRISTIANO es Doctor en Sociología por la Universidad Complutense de Madrid. Se desempeña como Investigador de carrera de CONICET (Consejo Nacional de Investigaciones Científicas y Tecnológicas) en el Centro de Estudios Avanzados de la Universidad Nacional de Córdoba (Argentina). Es Profesor Titular de Teoría Sociológica y Modernidad en la misma Universidad, y su campo de investigación es la teoría sociológica, con especial referencia a las teorías contemporáneas de la acción social. Ha publicado artículos en diversas revistas especializadas, RIS, REIS, RES, Inguruak y Papers entre las españolas, además del libro Lo social como institución imaginaria: Castoriadis y la teoría sociológica, en la editorial argentina EDUVIM.

\section{RECIBIDO: 01/12/09}

ACEPTADO:01/06/10

Publicado on-line: 25/02/2011 